\title{
Pathway Distribution Model for Solute Transport in Stratum Corneum
}

\author{
DANILA G. PETLIN ${ }^{1,2}$, MAKSYM RYBACHUK ${ }^{3,4}$ and YURI G. ANISSIMOV ${ }^{1,4 *}$ \\ ${ }^{1}$ School of Natural Sciences, Griffith University, 170 Kessels Road, Nathan QLD 4111, \\ Australia \\ 2 Tomsk Polytechnic University, 30 Lenin Avenue, Tomsk 634050, Russian Federation \\ ${ }^{3}$ School of Engineering, Griffith University, Engineering Drive, Southport QLD 4222, \\ Australia \\ ${ }^{4}$ Queensland Micro- and Nanotechnology Centre, Griffith University, Nathan QLD 4111, \\ Australia \\ This is the pre-peer reviewed version of the following article: FULL CITE, which has been published in final form \\ at [Link to final article]. Contributors are not required to remove preprints posted to not for profit preprint servers \\ prior to submission of the Contribution. \\ *Corresponding author:y.anissimov@griffith.edu.au
}

\begin{abstract}
One of the main functions of the skin is to reduce the amount of water evaporating from the surface of a human body with outermost layer of the epidermis, stratum corneum (SC), forming a barrier, which protects underlying tissue from dehydration. Empirical data obtained for water penetration in SC is normally analysed using mathematical models, among which the homogeneous membrane model is commonly employed to describe transport kinetics in SC. However, the homogeneous membrane model failed to fit simultaneously the experimental data for water permeation and desorption ${ }^{1}$, as the model does not account for a complex structure of SC and irregular distribution of corneocytes. Our previous work ${ }^{1}$ introduced a slow binding model that is more aligned with the true biological structure of SC. This report provides an alternative/additional model to both the homogeneous membrane and slow binding models and takes into account the distribution of effective pathways across SC for water transport.
\end{abstract}

Keywords: stratum corneum, water transport, diffusion modelling, skin reservoir effect, pathway distribution 


\section{INTRODUCTION}

Research efforts focused on studies of transdermal processes are increased annually as a way of developing new transdermal drug delivery systems for the pharmaceutical market, improving existing applications and designing new methods with enhanced capabilities. At present there is a variety of different mathematical models ${ }^{1-6}$ used to evaluate experimental data and predict the diffusion of substances, including water, through the human skin. Most of these mathematical models are based on the investigation of the rate-limiting barrier of the human body, stratum corneum (SC) ${ }^{7}$. These investigations involve measurement and systematization of different characteristics of solute transport in SC and the analysis of experimental data in an attempt to develop understanding about underlying functional processes in SC. At present there are two research approaches that used to investigate solute transport processes in SC. For solute media such as water, these are: a) transepidermal water loss and, b) water penetration and desorption studies in $\mathrm{SC}^{7-10}$. It was revealed ${ }^{1}$ that homogeneous membrane (HM) model showed a poor quality of regression between the obtained experimental data and the fitting functions for simultaneous water permeation and desorption processes in SC, therefore a model that accounted for heterogeneity of SC was implemented.

There are also models ${ }^{11,12}$, which represent with more detail real physiological structure of SC. Hitherto, in most cases, these models have been applied to describe the steady state kinetics and thus they are not well suited for time-dependent data especially for SC desorption experiments. To our knowledge, such detailed models have never been used for fitting time dependent experimental data. In the first instance, such models have too many parameters $^{12}$ (e.g. the actual dimensions of corneocytes, corneocytes thickness and their relative arrangement partitioning between lipids and corneocytes). The other reason is that the models mentioned could be too computationally demanding to be practical in fitting experimental data.

For these very reasons less complex and less numerically demanding models for solute transport are necessary. Commonly-used HM model and other transient models ${ }^{1,13}$ are convenient for the analysis of a transient solute transport through SC and have a potential to capture a detailed insight into the processes occurring in SC as it describes the solute transport close to the real biological environment. Such knowledge is important for transdermal drug delivery and other fields of research. 
In previous work ${ }^{1}$ we expanded the general understanding of water transport process through SC and interpreted the experimental results assuming slow diffusion of the solute into and out of corneocytes. It is well known that water in SC exists in both free and bound states $^{14,15}$. Also it was indicated ${ }^{16,17}$ that extended hydration in SC may contribute to the formation of large water pools leading to a slow equilibration within SC. These properties of SC are accounted for in the equilibration process between the bound and free states in a so-called slow binding (SB) model ${ }^{1,18}$. The SB model, while describing SC in more detail, is not mathematically demanding and has only two additional parameters compared to the HM model. Whereas, the SB model approach demonstrated a good fit with the observed experimental data, it does not indisputably mean that hypothesis used in the SB model accounts for a single, exclusive explanation of solute transport processes in SC. Therefore, it is important to re-evaluate and investigate these modelling approaches with unbiased scrutiny seeking to explain the observed experimental results using new paradigms and with an improved accuracy.

In our investigation we adapted brick-and-mortar structure representing SC and its' constituent corneocyte layers as shown schematically in Figure 1. It is well known that the SC barrier is composed of approximately 10 - 20 layers of corneocytes covering most parts of the surface of a human body ${ }^{7}$. The variability in the number of corneocyte layers accounts for a non-uniform thickness of SC within a small sampling area of the skin harvested for an experimental study (as shown in Figure 1). As corneocyte layers are known to be of different thickness, the intra-layer distances between constituent corneocyte layers vary as well, which overall adds to the complexity of describing solute transport pathways in SC with any degree of certainty. In addition, water is known to diffuse straight through corneocyte layers (transcellular route) as shown by works of Kasting et al. ${ }^{19}$; this instance is schematically illustrated in Figure 1. Therefore, the discussion concerning exact contributions of extracellular and transcellular solute diffusion pathways in SC is still open $^{20}$ and the possible solutions offered through this study will further add to the knowledge base regarding transport pathways heterogeneity in SC. 


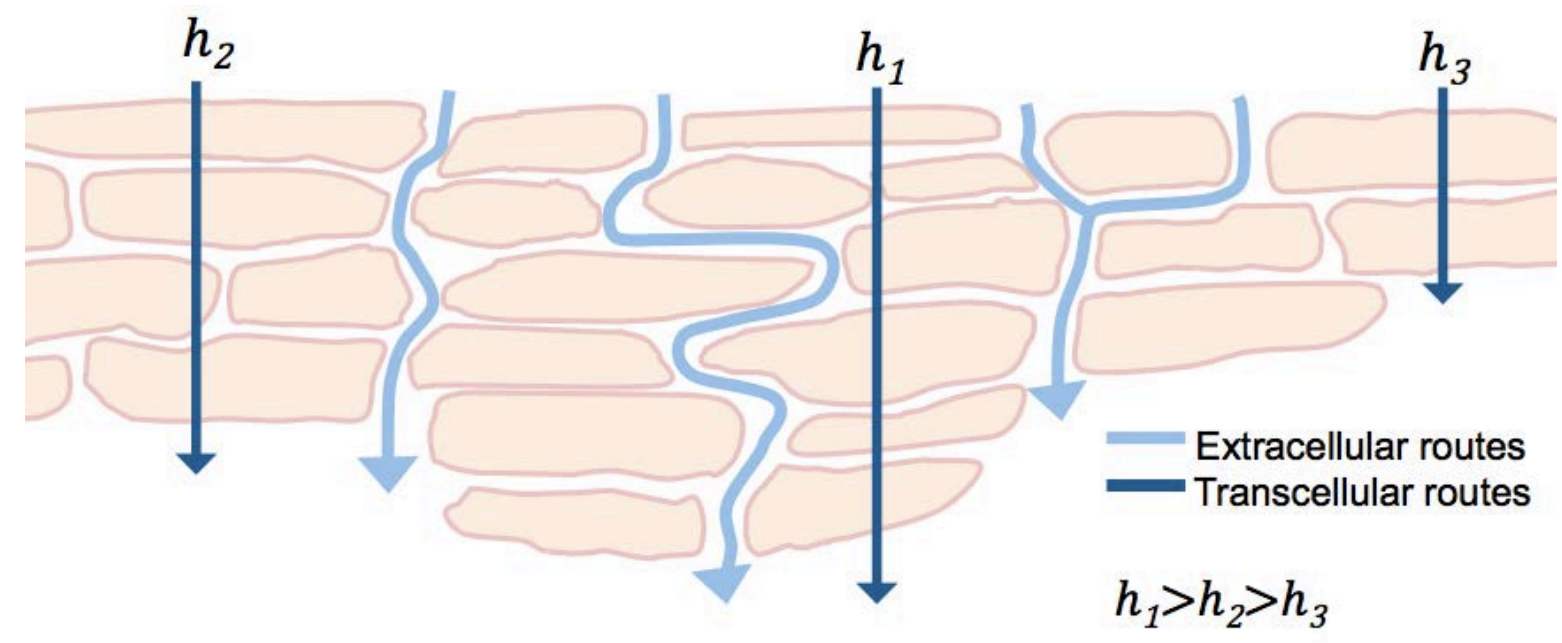

Figure 1. Schematic representation of stratum corneum with corneocytes of different thickness and of different dimensions and arrangements leading to variable transcellular and extracellular solute pathways lengths distribution.

As shown in Figure 1 the solute may proceed straight through corneocytes (transcellular route) or through lipid phases (extracellular route). The length of each pathway may vary due to the number of corneocyte layers solute goes through, the dimensions of corneocytes and their arrangement. For this reason, we named the new model - the pathway distribution (PD) model. It needs to be stressed that the PD model applies explicitly for "independent" transcellular pathways of different lengths, however extracellular pathways may share some common paths (see Figure 1), therefore the model can only be implicitly relevant for extracellular pathways.

In addition, the SB and PD models combined together were analysed. The practical importance of this work stands in improving the understanding about the potential mechanisms associated with the skin reservoir effect ${ }^{21}$ by taking into account various physiological aspects of solute transport which are inherently present in SC. 


\section{Experimental data}

The experimental data was adapted from our previous work ${ }^{1}$ including the type of SC samples used together with desorption and permeation experiment particulars. Numerical inversions of Laplace domain solutions and non-linear regressions were carried out using the program Scientist ${ }^{\circledR}$ (MicroMath, USA) and a custom-written program in Python programming language. Weighting of $1 / y_{\text {observed }}$ was used for nonlinear regressions.

\section{Model Description}

The proposed PD model aims to expand the utility of the simple the HM model by taking into account that solute diffuses through SC following pathways of variable lengths. Many factors define the heterogeneity of SC pathways, such as the variability of corneocyte layers (i.e. SC thickness), layer dimensions and layer arrangement. Assuming that none of these multiple factors play a dominant role and based on the central limit theorem (i.e. averages of independent random variables drawn from any distributions are normally distributed) allow us to adopt the normal distribution for pathway lengths in SC. The uniform distribution of SC lengths was also selected as an additional, basic comparative pilot model to assess the sensitivity of the PD model to the selection of the distribution. Therefore solute pathways in SC were considered to have a) the uniform or, b) the normal distribution as illustrated in Figure 2, with $h_{i}$ corresponding to the length of an ith pathway. The approach is similar to modelling of solute transport in liver where there is a variety of distribution functions assigned to various sinusoidal lengths and solute velocities ${ }^{22,23}$.

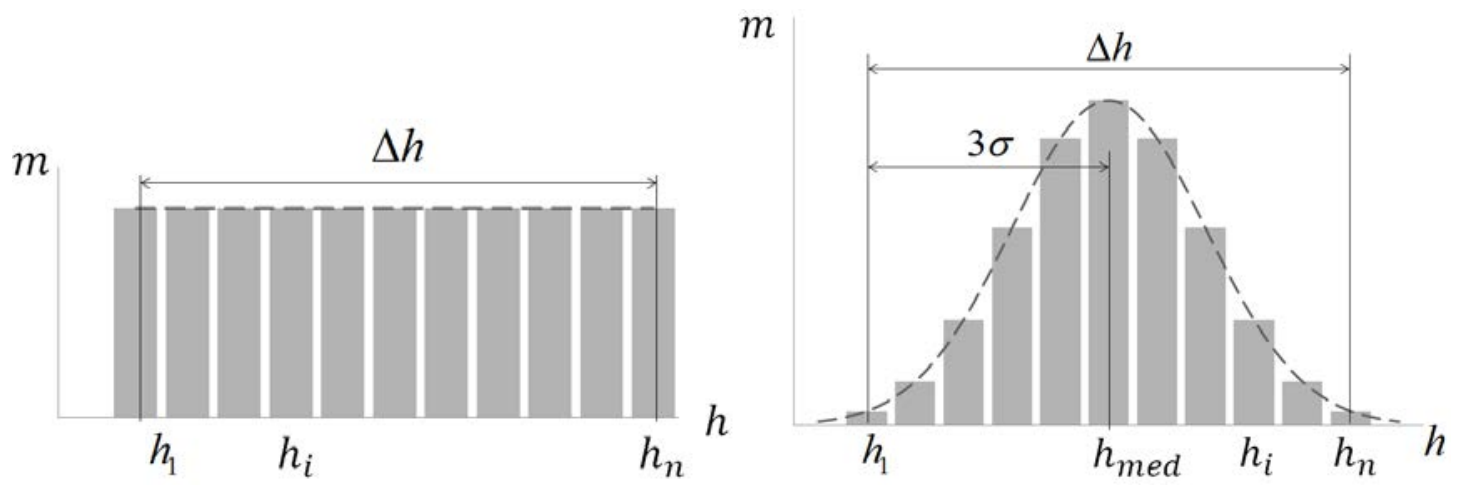


(a)

Figure 2. Probability density function $m\left(h_{i}\right)$ of solute pathways following a) the uniform and b) the normal distribution of solute pathway lengths, $h_{i}$, and their respective discretisations.

The amount of solute penetrated and solute desorbed can be represented as a sum:

$$
Q(t)=\sum_{i} Q_{i}\left(h_{i}, t\right)
$$

where $h_{i}$ is the length of an $i$-pathway and the summation is performed for all pathways. In order to calculate the sum we used the Laplace domain solution:

$$
\hat{Q}(s)=\sum_{i} \hat{Q}_{i}\left(h_{i}, s\right)
$$

where $s$ is the Laplace variable and $\wedge$ over functions denotes its Laplace transform. For each of the pathway length, $h_{i}$, the amount of solute penetrated $\left(\hat{Q}_{p}\left(h_{i}, s\right)\right)$ and the amount of solute desorbed $\left(\hat{Q}_{d}\left(h_{i}, s\right)\right)$ can be expressed ${ }^{1}$ as:

$$
\begin{array}{r}
\hat{Q}_{p}\left(h_{i}, s\right)=\frac{m\left(h_{i}\right)}{s^{2} t_{d i}} \frac{\sqrt{s t_{d i}}}{\sinh \sqrt{s t_{d i}}} \\
\hat{Q}_{d}\left(h_{i}, s\right)=\frac{m\left(h_{i}\right)}{s} \frac{2 \tanh \left(\frac{1}{2} \sqrt{s t_{d i}}\right)}{\sqrt{s t_{d i}}}
\end{array}
$$

where $m\left(h_{i}\right)$ is the amount of water associated with an ith pathway after sorption, and $t_{d i}$ ( $=h_{i}^{2} / D$, where $D$ is the diffusion coefficient, assumed to be the same for all pathways) is 
the characteristic time for the given $h_{i}$. Notably, Eq. (3) and (4) are based on employing the HM solutions for each pathway length.

The probability density function, $m\left(h_{i}\right)$ defines each $h_{i}$ distribution. In the PD model we assume that pathways lengths can vary within the range of $\Delta h$ as shown in Figure 2. For the sake of approximating the normal distribution it was assumed that $\Delta h=6 \sigma$, where $\sigma$ is the standard deviation. The value for $\Delta h$ was selected to represent the wide range of the normal distribution function. Notably, hair follicles and other skin appendages can potentially contribute to very short pathway lengths, though the fully hydrated SC skin appendages are normally closed during in vitro experiments due to the SC being swollen. To simplify the calculation of the sum, Eq. (2), we discretise various pathways with close $h_{i}$ values into $n$ groups. After discretisation (as shown in Fig. 2), $m\left(h_{i}\right)$ can be replaced with $m\left(h_{i}\right)=\frac{m_{0}}{n}$ for uniform and $m\left(h_{i}\right)=c e^{-\frac{\left(h_{i}-h_{\text {med }}\right)^{2}}{2 \sigma^{2}}}$ for normal pathways distribution $\left(m_{0}\right.$ is a total amount desorbed), with $c$ as the normalising coefficient, which depends on discretisation number, $n$, and on pathway distribution range, $\Delta h$, giving $\sum_{i=1}^{n} m\left(h_{i}\right)=m_{0}$.

In this work the proposed PD model was compared with the SB model ${ }^{1}$ to describe solute transport in SC. We also considered a combined PD/SB model, which takes into account solute slow binding process and pathways distribution. For the latter case Eq. (3) and (4) need to be amended as:

$$
\begin{gathered}
\hat{Q}_{p}\left(h_{i}, s\right)=\frac{m_{i}}{s^{2} t_{d i}} f_{u} \frac{\sqrt{g(s) t_{d i}}}{\sinh \sqrt{g(s) t_{d i}}} \\
\hat{Q}_{d}\left(h_{i}, s\right)=\frac{m_{i}}{s}\left[f_{u}+\frac{k_{\text {off }}\left(1-f_{u}\right)}{s+k_{\text {off }}}\right] \frac{2 \tanh \left(\frac{1}{2} \sqrt{g(s) t_{d i}}\right)}{\sqrt{g(s) t_{d i}}}
\end{gathered}
$$

where $k_{\text {on }}$ and $k_{\text {off }}$ are the rate constants for slow binding, $f_{u}=\left(1+k_{\text {on }} / k_{\text {off }}\right)^{-1}$ is an unbound fraction and $g(s)$ given by $g(s)=s+k_{\text {on }}-k_{\text {on }} k_{\text {off }} /\left(s+k_{\text {off }}\right)$. 


\section{RESULTS AND DISCUSSION}

The experimental data for the amount of water desorbed from SC (represented as squares, Fig. 3) and the amount of water penetrated SC (represented as circles, Fig. 3) as a function of time is shown in Figure 3. Since the experimental data is characteristic of one SC sample harvested from one subject the data analysis of desorption and penetration experiments necessitate the application of identical fitting parameters. Therefore, simultaneous fitting of desorption and penetration data was performed using the PD model. The characteristic time, $t_{d}\left(=h_{\text {med }}^{2} / D\right)$, and the pathway distribution standard deviation, $\sigma$, were chosen as fitting parameters. The fitted curves to data points in Figure 3 represent the comparison between the uniform and normal distributions assumed in PD model. Parameter estimates of $t_{d}=327 \pm 17 \mathrm{~min}$ and $\sigma=0.308 \pm 0.002$ were obtained for the normal distribution, $t_{d}=220 \pm 9$ min and $\sigma=0.243 \pm 0.009$ were obtained for the uniform distribution (with $m_{0}$ fixed to $1.55 \mathrm{mg} / \mathrm{cm}^{2}$ ). Notably, there is only a minor difference observed concerning the quality of regression for two distributions (see Fig. 3). The value for model selection criterion - the parameter similar to Akaike information criterion ${ }^{24}$ for the normal distribution was found to be 5.1, whereas the value of 4.7 was obtained for the uniform distribution. The higher value for model selection criterion served as justification for selecting the normal distribution for further data analysis employed in PD model. The other reason to select the normal distribution is that it approximates many natural phenomena known ${ }^{25}$. 


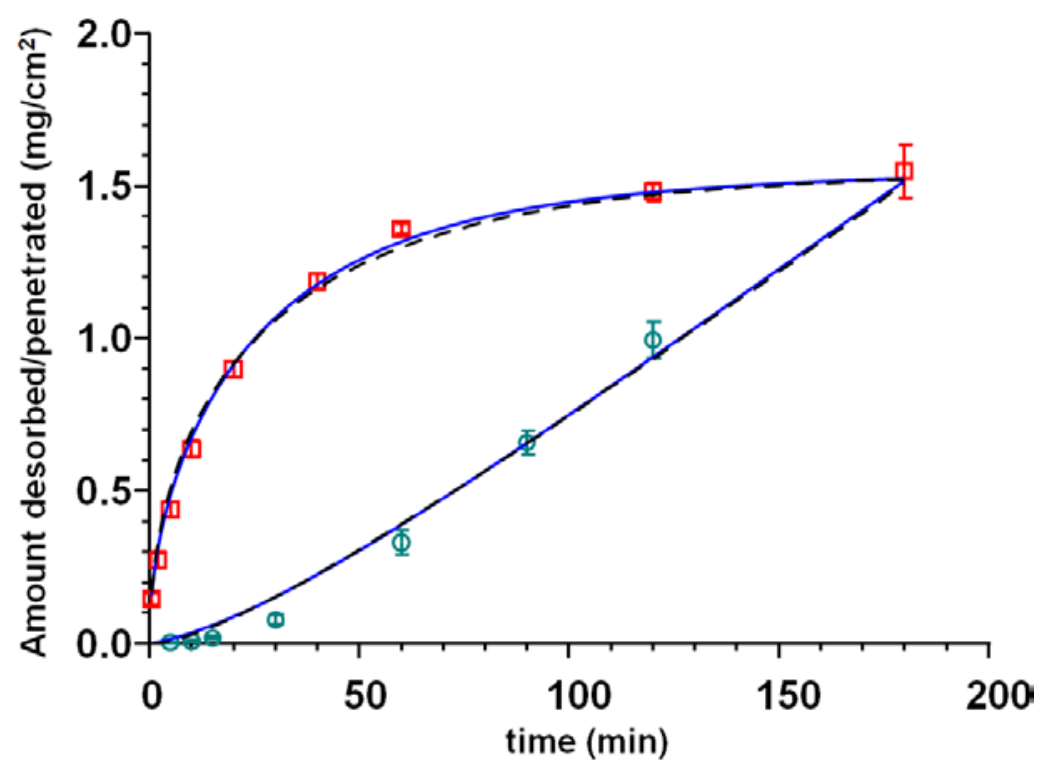

Figure 3. Experimental data showing water desorption (squares) and water penetration (circles) profiles in SC and their simultaneous regression profiles for uniform pathway distribution model (dashed line) and the normal pathway distribution model (solid line).

Figure 4 shows the results of simultaneous fitting of the experimental data for desorption and penetration processes using a) HM model, b) SB model and c) normal PD model. Evidently, simultaneous fitting of the experimental data using the HM model ( $t_{d}=151 \pm 12 \mathrm{~min}, m_{0}=1.37 \pm 0.05 \mathrm{mg} / \mathrm{cm}^{2}$ ) produce a poor regression quality fit (see dashed line, Fig. 4) with a non-random distribution of residuals, which indicates that a more complex fitting model should be used. It is worth noting that the HM model fits separately the desorption and penetration data well, but this leads to markedly different values of $t_{d}$ indicating the limitation of the HM model. The proposed PD model definitely solves the poor regression characteristics of the HM model and provides a suitable fit. The results reported in Figure 4 are obtained for discretisation number $n=9$, however, the increase of an $n$ number did not afford any significant improvements of the regression. The PD model shows a slight deviation as compared to the SB model limited to the time range from 40 to 120 minutes. The most significant difference in fit between the HM, SB and PD models is observed at 60 minutes for the desorption profile. Also, the quality of the SB model fit at early stage of penetration process (first 20 minutes) is found to be closer to experimental data points than the fit of the PD model. The observed discrepancy suggests that the PD model is unable to fully explain the transport processes within the SC during water 
desorption and the attributes of the SB model are required to fully explain the experimental data.

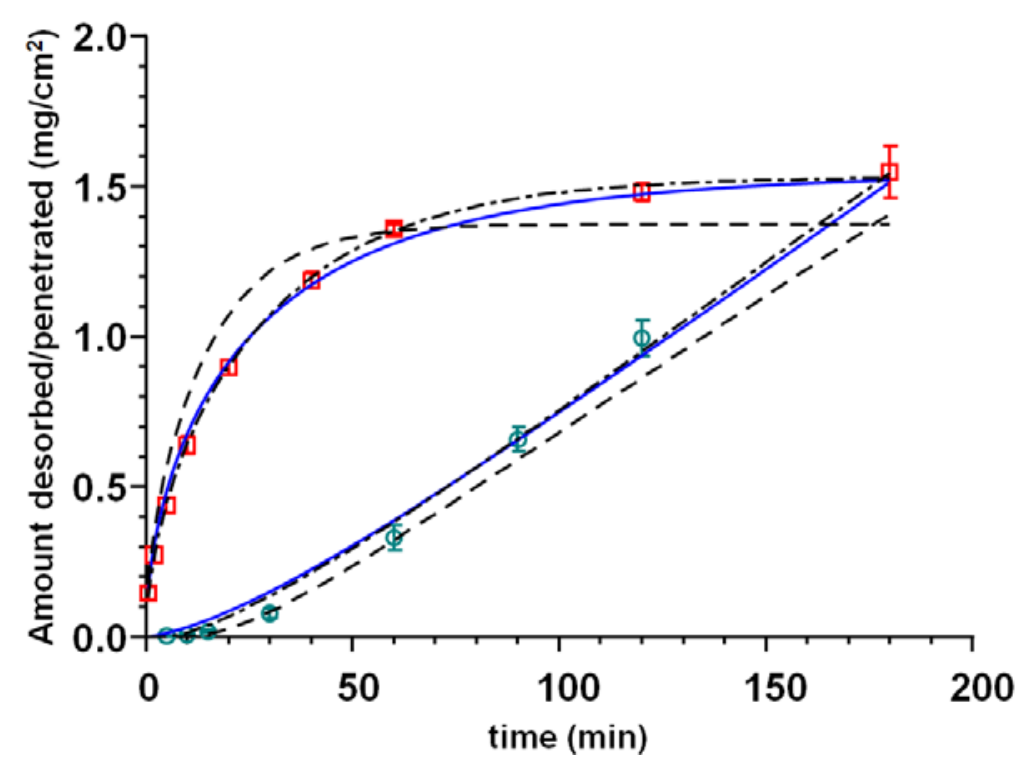

Figure 4. Experimental data showing water desorption (squares) and water penetration (circles) profiles in stratum corneum and their simultaneous regression profiles for a) the homogeneous membrane model (dashed line), b) the slow binding model (dot-dashed line) and, c) the normal pathway distribution model (solid line).

The combined model that included attributes of the PD and SB models which accounts for slow binding characteristics of solute in SC and the normal distribution of solute pathways in SC was applied to the experimental data. The combined model, however, did not provide a noticeable improvement in the fit (Table 1). The fitting of the combined model was performed using standard deviation value $\sigma=0.308$ which was initially fixed to that obtained in the PD model regression. Another reason why this value was used is that it appears to correspond to the reported variation of corneocyte layers in the $\operatorname{trunk}^{7}(13 \pm 4$ corneocyte layers) assuming that pathway lengths distribution is only due to the variation of the number of corneocytes layers in SC. The value for the model selection criterion for the SB model was 5.7, whereas the value of 5.4 was found for the combined model. The lower value of model selection criterion for combined model is due to using more parameters for fitting $\left(k_{o n}, k_{o f f}, \sigma, t_{d}\right)$. 
Table 1. The comparison of investigated models

\begin{tabular}{|l|c|c|c|c|c|}
\hline Model & $t_{d}$, min & $\begin{array}{c}\text { Mean } \\
\text { desorption } \\
\text { time, min }\end{array}$ & $\sigma$ & $k_{\text {on, }} k_{\text {off }}$ & $\begin{array}{c}\text { Model } \\
\text { selection } \\
\text { criterion }\end{array}$ \\
\hline Homogeneous membrane & $151 \pm 12$ & 13 & N/A & N/A & 3.14 \\
\hline Slow binding & $69 \pm 7$ & 26 & N/A & $0.052,0.042$ & 5.69 \\
\hline Normal pathway distribution & $327 \pm 17$ & 27 & 0.31 & N/A & 5.05 \\
\hline Uniform pathway distribution & $220 \pm 9$ & 18 & 0.24 & N/A & 4.70 \\
\hline Combined (normal pathway & $110 \pm 9$ & 31 & 0.27 & $0.018,0.026$ & 5.38 \\
distribution and slow binding) & & 28 & 0.31 & $0.015,0.026$ & 5.39 \\
\hline
\end{tabular}

Further analysis of the combined model when both slow equilibration mechanisms and the normal pathway distribution are taken into consideration demonstrated that binding/unbinding rate constants for solute in SC change when the standard deviation is varied (Table 1). The change of $k_{\text {on }}$ and $k_{\text {off }}$ for the case of combined model as compared to binding rates for the SB model alone demonstrates that the SB model parameters are sensitive to the assumption of the pathways lengths distribution. The comparison of $t_{d}$ values also demonstrates the strong sensitivity of the combined model to including the pathway distribution parameters (Table 1). Mean desorption time $(M D T)^{1}$

$$
M D T=\frac{t_{d}}{12 f_{u}}+\frac{1-f_{u}}{k_{o f f}}
$$

was introduced in Table 1 as a useful parameter to describe, gauge and compare desorption kinetics in SC. It demonstrates that though the parameter $t_{d}$ is quite different for the two models, a more physically relevant $M D T$ value is close for the SB and PD models.

The main utility of the presented PD model is that it demonstrates an alternative/additional extension to the HM model that is based on the SC physiology and can help better interpret parameters of the SB model. Although the SB model fitted the data for water penetration and desorption very well, it is not possible to ascertain unambiguously what is the "physiological source" of the slow binding rate constants. We believe that the constants are most likely determined by the slow partitioning into corneocytes, but some contribution to those rates from other factors, such as the variable pathways, are possible. Results presented in Table 1 indeed demonstrate that the slow binding rate constants are sensitive to the change of the standard deviation $(\sigma)$ which describes the pathways length distribution. Furthermore, while for the case of water experimental data the combined model approach 
did not demonstrate the statistically significant regression improvement for solute transport data in comparison with the SB model, in future the analysis of experimental data for other solutes may demonstrate the utility of the PD model and especially the combined model.

\section{CONCLUSIONS}

In this work we formulated a new pathway distribution model to describe solute transport though SC. The PD model was applied to fit the experimental data for simultaneous water penetration and desorption and the fitting results were compared to the previously published mathematical model that considered the effect of slow equilibration between bound and unbound water in SC. The regression of the data obtained using the new pathway distribution model showed a good fit, however the obtained fitting results did not exceed quantitatively the results obtained previously using the slow binding model. Therefore, we concur that the slow binding mechanisms for water transport associated with the skin reservoir effect explain the experimental data in a more detailed way. The combination of slow equilibration and the normal pathway distribution approaches was found to be insufficiently improving the fitting for water transport experimental data in SC. However, the combined model approach may be applicable for the analysis of solute transport in SC for solute media other than water. Notably, the normal PD model proposed in this work demonstrates a significantly better fit of the experimental data as compared to homogeneous membrane model proposed earlier ${ }^{1}$. Further experimental investigation of solute transport in SC is required to determine the extent of pathway non-uniformity in SC with higher resolution and enhanced precision.

\section{ACKNOWLEDGEMENTS}

MR acknowledges Sir Samuel Griffith Lectureship Program and research seed funding from the Griffith School of Engineering. 


\section{REFERENCES}

1. Anissimov YG, Roberts MS 2009. Diffusion modeling of percutaneous absorption kinetics: 4. Effects of a Slow Equilibration Process Within Stratum Corneum on Absorption and Desorption Kinetics. J Pharm Sci 98:772-781.

2. Couto A, Fernandes R, Cordeiro MNS, Reis SS, Ribeiro RT, Pessoa AM 2014. Dermic diffusion and stratum corneum: A state of the art review of mathematical models. Journal of Controlled Release 177(0):74-83.

3. Anissimov YG, Jepps OG, Dancik Y, Roberts MS 2013. Mathematical and pharmacokinetic modelling of epidermal and dermal transport processes. Adv Drug Deliv Rev 65:169-190.

4. Anissimov YG, Watkinson A 2013. Modelling skin penetration using the laplace transform technique. Skin Pharmacol Physiol 26(4-6):286-294.

5. Anissimov YG, Roberts MS 1999. Diffusion modeling of percutaneous absorption kinetics: 1. Effects of flow rate, receptor sampling rate and viable epidermal resistance for a constant donor concentration. J Pharm Sci-Us 88(11):1201-1209.

6. Kasting GB 2001. Kinetics of finite dose absorption through skin 1. Vanillylnonanamide. J Pharm Sci 90(2):202-212.

7. Ya-Xian Z, Suetake T, Tagami H 1999. Number of cell layers of the stratum corneum in normal skin - relationship to the anatomical location on the body, age, sex and physical parameters. Arch Dermatol Res 291(10):555-559.

8. Gay R, Swiderek M, Nelson D, Ernesti A 1992. The living skin equivalent as a model in vitro for ranking the toxic potential of dermal irritants. Toxicology in vitro : an international journal published in association with BIBRA 6(4):303-315.

9. Friberg SE, Kayali I, Beckerman W, Rhein LD, Simion A 1990. Water Permeation of Reaggregated Stratum Corneum with Model Lipids. J Investig Dermatol 94(3):377-380.

10. Kalia YN, Alberti I, Sekkat N, Curdy C, Naik A, Guy RH 2000. Normalization of stratum corneum barrier function and transepidermal water loss in vivo. Pharmaceut Res 17(9):1148-1150.

11. Wang TF, Kasting GB, Nitsche JM 2006. A multiphase microscopic diffusion model for stratum corneum permeability. I. Formulation, solution, and illustrative results for representative compounds. J Pharm Sci 95(3):620-648.

12. Frasch HF, Barbero AM 2003. Steady-state flux and lag time in the stratum corneum lipid pathway: results from finite element models. J Pharm Sci 92(11):2196-2207.

13. Frasch HF 2002. A random walk model of skin permeation. Risk Anal 22(2):265-276.

14. Scheuplein RJ, Morgan LJ 1967. "Bound water" in keratin membranes measured by a microbalance technique. Nature 214(87):456-458.

15. Walkley K 1972. Bound water in stratium corneum measured by differential scanning calorimetry. J Invest Dermatol 59(3):225-227.

16. Warner RR, Stone KJ, Boissy YL 2003. Hydration disrupts human stratum corneum ultrastructure. J Invest Dermatol 120(2):275-284.

17. Bouwstra JA, de Graaff A, Gooris GS, Nijsse J, Wiechers JW, van Aelst AC 2003. Water distribution and related morphology in human stratum corneum at different hydration levels. Journal of investigative dermatology 120(5):750-758.

18. Frasch HF, Barbero AM, Hettick JM, Nitsche JM 2011. Tissue binding affects the kinetics of theophylline diffusion through the stratum corneum barrier layer of skin. J Pharm Sci 100(7):2989-2995.

19. Kasting GB, Barai ND, Wang TF, Nitsche JM 2003. Mobility of water in human stratum corneum. J Pharm Sci 92(11):2326-2340. 
20. Xiao P, Imhof RE 2012. Two dimensional finite element modelling for dynamic water diffusion through stratum corneum. International Journal of Pharmaceutics 435(1):88-92.

21. Roberts MS, Cross SE, Anissimov YG 2004. Factors affecting the formation of a skin reservoir for topically applied solutes. Skin Pharmacology and Applied Skin Physiology 17:3-16.

22. Anissimov YG, Bracken AJ, Roberts MS 1997. Interconnected-tubes model of hepatic elimination. J Theor Biol 188(1):89-101.

23. Bass L, Robinson P, Bracken AJ 1978. Hepatic Elimination of Flowing Substrates Distributed Model. J Theor Biol 72(1):161-184.

24. Sakamoto Y, Ishiguro M, Kitagawa G 1986. Akaike information criterion statistics. Dordrecht, The Netherlands: D Reidel.

25. Limpert E, Stahel WA, Abbt M 2001. Log-normal distributions across the sciences: Keys and clues. Bioscience 51(5):341-352. 